\title{
New identities involving Bernoulli, Euler and Genocchi numbers
}

\author{
Su Hu${ }^{1}$, Daeyeoul Kim² and Min-Soo Kim ${ }^{3 *}$
}

"Correspondence:

mskim@kyungnam.ac.kr

${ }^{3}$ Division of Cultural Education,

Kyungnam University, 7

(Woryeong-dong)

kyungnamdaehak-ro,

Masanhappo-gu, Changwon-si, Gyeongsangnam-do 631-701,

Republic of Korea

Full list of author information is

available at the end of the article

\author{
Abstract \\ Using $p$-adic integral, many new convolution identities involving Bernoulli, Euler and \\ Genocchi numbers are given. \\ MSC: $11 \mathrm{~B} 68 ; 11 \mathrm{~S} 80$ \\ Keywords: Bernoulli numbers; Euler numbers; sums of products; fermionic $p$-adic \\ integral; Volkenborn integral
}

\section{Introduction}

The study of the identities involving Bernoulli numbers and polynomials, Euler numbers and polynomials has a long history. More than 250 years ago, Euler discovered the following sums of products identity involving Bernoulli numbers:

$$
\sum_{l=1}^{n-1}\left(\begin{array}{c}
2 n \\
2 l
\end{array}\right) B_{2 l} B_{2 n-2 l}=-(2 n+1) B_{2 n} \quad(n>1)
$$

(see [1]).

This identity has been extended by many authors in different directions (see [2-21]). Recently, Kim and Hu [22] obtained an analogy of Euler's sums of products identity for Apostol-Bernoulli numbers.

In 1978, Miki [23] obtained the following well-known identity, which is now known as Miki's identity:

$$
\sum_{k=2}^{n-2} \frac{B_{k} B_{n-k}}{k(n-k)}-\sum_{k=2}^{n-2}\left(\begin{array}{l}
n \\
k
\end{array}\right) \frac{B_{k} B_{n-k}}{k(n-k)}=2 H_{n} \frac{B_{n}}{n}
$$

for every $n=4,5, \ldots$, where $H_{n}=1+1 / 2+\cdots+1 / n$.

In 1997, Matiyasevich [24] found another identity of this type

$$
(n+2) \sum_{k=2}^{n-2} B_{k} B_{n-k}-2 \sum_{k=2}^{n-2}\left(\begin{array}{c}
n+2 \\
k
\end{array}\right) B_{k} B_{n-k}=n(n+1) B_{n}
$$

for any $n=4,5, \ldots$.

In 2004, Dunne and Schubert [25] obtained the convolution identities for sums of products of Bernoulli numbers motivated by the role of these identities in quantum field theory

\section{Springer}

c) $2013 \mathrm{Hu}$ et al: licensee Springer. This is an Open Access article distributed under the terms of the Creative Commons Attribution License (http://creativecommons.org/licenses/by/2.0), which permits unrestricted use, distribution, and reproduction in any medium, provided the original work is properly cited. 
and string theory. In 2006, Crabb [26] showed that Gessel's generalization of Miki's identity [27] is a direct consequence of a functional equation for the generating function. During the same year, Sun and Pan [28] established two general identities involving Bernoulli and Euler polynomials, which imply both Miki and Matiyasevich's identities.

According to statement by Cohen in the first paragraph of [29, Chapter 11], the $p$-adic functions with nice properties are powerful tools for studying many results of classical number theory in a straightforward manner, for instance, strengthening of almost all the arithmetic results on Bernoulli numbers.

For example, in Exercises 2 and 3 of [29, Chapter 11], Cohen demonstrated a method to prove the following reciprocity formula for Bernoulli numbers using $p$-adic integral:

$$
m ! \sum_{j=0}^{m} \frac{B_{m-j}}{(m-j) !} \frac{B_{n+j+1}}{(j+1) !}+n ! \sum_{j=0}^{n} \frac{B_{n-j}}{(n-j) !} \frac{B_{m+j+1}}{(j+1) !}=-B_{m+n}
$$

(see Exercise 3(c) of [29, Chapter 11]).

Recently, using $p$-adic integral, Kim et al. [8] proved several identities on Bernoulli and Euler numbers. More comprehensive coverage can be found in the monographs by Chio et al. [2], Kim et al. [4], Kim et al. [5], Kim et al. [6], Kim et al. [7], Kim and Kim [9], Kim et al. [13], Lee and Kim [14].

In this paper, following the methods of [8], we shall further provide many new convolution identities involving Bernoulli, Euler and Genocchi numbers.

Let $B_{n}(x), E_{n}(x)$ and $G_{n}(x)$ be the $n$th Bernoulli, Euler and Genocchi polynomials, respectively. In what follows, we use $E_{n}$ to denote the special value of $E_{n}(x)$ at 0 , that is, $E_{n}=E_{n}(0)$.

Let $\delta_{i, j}$ be the Kronecker symbol defined by $\delta_{i, i}=1$ and $\delta_{i, j}=0$ for $i \neq j$.

In fact, the following identities are shown in this paper:

$$
\begin{aligned}
& \sum_{l=0}^{2 n+1}\left(\begin{array}{c}
2 n+1 \\
l
\end{array}\right) B_{2 n-l+1} E_{l}=-\delta_{0, n} \quad \text { (Theorem 3.3), } \\
& \sum_{k=0}^{2 m+1} \sum_{l=0}^{2 n+2}\left(\begin{array}{c}
2 m+1 \\
k
\end{array}\right)\left(\begin{array}{c}
2 n+2 \\
l
\end{array}\right) B_{2 m-k+1} E_{2 n-l+2} E_{k+l}=0 \quad \text { (Theorem 3.4), } \\
& \sum_{k=0}^{2 m+2} \sum_{l=0}^{2 n+1}\left(\begin{array}{c}
2 m+2 \\
k
\end{array}\right)\left(\begin{array}{c}
2 n+1 \\
l
\end{array}\right) B_{2 m-k+2} E_{2 n-l+1} E_{k+l}=2 B_{2 m+2} E_{2 n+1} \quad \text { (Theorem 3.4) } \\
& \sum_{l=0}^{2 n+2}\left(\begin{array}{c}
2 n+2 \\
l
\end{array}\right) E_{2 n-l+2}\left(E_{l}-2 E_{l+1}\right)=0 \quad(\text { Corollary 3.5) } \\
& \sum_{k=0}^{2 m+1}\left(\begin{array}{c}
2 m+1 \\
k
\end{array}\right) B_{2 m-k+1}\left(E_{k+2}-E_{k+1}\right)=0 \quad(\text { Corollary 3.5), } \\
& \sum_{k=0}^{2 m}\left(\begin{array}{c}
2 m \\
k
\end{array}\right) B_{2 m-k}\left(E_{k}-2 E_{k+1}\right)=2 B_{2 m} \quad(\text { Corollary 3.5), } \\
& \sum_{l=0}^{2 n+1}\left(\begin{array}{c}
2 n+1 \\
l
\end{array}\right) E_{2 n-l+1}\left(E_{l}-6 E_{l+1}+6 E_{l+2}\right)=2 E_{2 n+1} \quad \text { (Corollary 3.5), }
\end{aligned}
$$




$$
\begin{aligned}
& \sum_{l=0}^{2 n+1}\left(\begin{array}{c}
2 n+1 \\
l
\end{array}\right) E_{2 n-l+1} E_{l}=2 E_{2 n+1} \quad \text { if } n \in \mathbb{N}(\operatorname{Remark} 3.6) \\
& \sum_{i=0}^{\alpha}(-1)^{i} B_{n}^{(\alpha)}(i)=\left\{\begin{array}{lll}
\sum_{l=0}^{n}\left(\begin{array}{c}
n \\
l
\end{array}\right) B_{n-l}^{(\alpha)} E_{l} & \text { if } n+\alpha \equiv 0 & (\bmod 2), \\
0 & \text { if } n+\alpha \neq 0 & (\bmod 2)(\text { Theorem 4.2) }
\end{array}\right. \\
& \sum_{i=0}^{\alpha}(-1)^{i} E_{n}^{(\alpha)}(i)=\left\{\begin{array}{lll}
\sum_{l=0}^{n}\left(\begin{array}{c}
n \\
l
\end{array}\right) E_{n-l}^{(\alpha)} E_{l} & \text { if } n+\alpha \equiv 0 & (\bmod 2), \\
0 & \text { if } n+\alpha \neq 0 & (\bmod 2)(\text { Theorem 4.3) }
\end{array}\right. \\
& \sum_{i=0}^{\alpha}(-1)^{i} B_{m}^{(\alpha)}(i) E_{n}^{(\alpha)}(i) \\
& =\left\{\begin{array}{lc}
\sum_{k=0}^{m} \sum_{l=0}^{n}\left(\begin{array}{c}
m \\
k
\end{array}\right)\left(\begin{array}{c}
n \\
l
\end{array}\right) B_{m-k}^{(\alpha)} E_{n-l}^{(\alpha)} E_{k+l} & \text { if } m+n+\alpha \equiv 0 \quad(\bmod 2), \\
0 & \text { if } m+n+\alpha \neq 0 \quad(\bmod 2) \\
(\text { Theorem 4.4) }
\end{array}\right. \\
& \sum_{i=0}^{\alpha}(-1)^{i} B_{m}^{(\alpha)}(i) G_{n}^{(\alpha)}(i) \\
& =\left\{\begin{array}{lll}
\sum_{k=0}^{m} \sum_{l=0}^{n}\left(\begin{array}{c}
m \\
k
\end{array}\right)\left(\begin{array}{c}
n \\
l
\end{array}\right) B_{m-k}^{(\alpha)} G_{n-l}^{(\alpha)} E_{k+l} & \text { if } m+n \equiv 0 & (\bmod 2), \\
0 & \text { if } m+n \neq 0 & (\bmod 2)(\text { Section 5). }
\end{array}\right.
\end{aligned}
$$

This paper is organized as follows. In the next section, we recall the fundamental results between $p$-adic integral and Bernoulli and Euler numbers. Then using these results we prove new identities (identities (1.2), (1.3), (1.4), (1.5), (1.7), (1.8), (1.9)) involving Bernoulli and Euler numbers in Section 3, prove new identities (identities (1.10), (1.11), (1.12)) involving higher-order Bernoulli and Euler numbers and polynomials in Section 4, and also prove a new identity (identity (1.13)) involving both Bernoulli, Euler and Genocchi numbers in the final section.

\section{2 p-adic analysis}

In this section, we recall the fundamental results between $p$-adic integral and Bernoulli and Euler numbers, and we see that the properties of $p$-adic integrals may imply most wellknown facts on Bernoulli numbers and polynomials, Euler numbers and polynomials.

We assume $p$ is an odd prime number. The symbols $\mathbb{Z}_{p}, \mathbb{Q}_{p}$ and $\mathbb{C}_{p}$ denote the rings of $p$-adic integers, the field of $p$-adic numbers and the field of $p$-adic completion of the algebraic closure of $\mathbb{Q}_{p}$, respectively. $\mathbb{N}$ denotes the set of natural numbers and $\mathbb{N}_{0}$ denotes $\mathbb{N} \cup\{0\}$.

Let $U D\left(\mathbb{Z}_{p}\right)$ be the space of uniformly (or strictly) differentiable function on $\mathbb{Z}_{p}$. Then the Volkenborn integral of $f$ is defined by

$$
I(f(z))=\int_{\mathbb{Z}_{p}} f(z) d \mu(z)=\lim _{N \rightarrow \infty} \frac{1}{p^{N}} \sum_{a=0}^{p^{N}-1} f(a)
$$

and this limit always exists when $f \in U D\left(\mathbb{Z}_{p}\right)$ (see [30, p.264]). For such functions we have

$$
I\left(f_{1}\right)-I(f)=f^{\prime}(0),
$$


where $f^{\prime}(0)=\left.(\mathrm{d} f(z) / \mathrm{d} z)\right|_{z=0}$ and $f_{1}(z)=f(z+1)$. By (2.2), we have the following Volkenborn $p$-adic integral representation of the generating function of Bernoulli polynomials $B_{n}(x)$ :

$$
I\left(e^{(x+z) t}\right)=\left(\frac{t}{e^{t}-1}\right) e^{x t}=\sum_{n=0}^{\infty} B_{n}(x) \frac{t^{n}}{n !}
$$

which is equivalent to

$$
I\left((x+z)^{n}\right)=B_{n}(x)
$$

(see [12])

Setting $x=0$ in (2.4), we obtain the Volkenborn $p$-adic integral representation of the $n$th Bernoulli numbers

$$
B_{n}=B_{n}(0)=I\left(z^{n}\right)
$$

(see $[12,30])$.

Thus from the binomial theorem and (2.4), we get

$$
B_{n}(x)=I\left((x+z)^{n}\right)=\sum_{l=0}^{n}\left(\begin{array}{l}
n \\
l
\end{array}\right) x^{l} I\left(z^{n-l}\right)=\sum_{l=0}^{n}\left(\begin{array}{l}
n \\
l
\end{array}\right) x^{l} B_{n-l},
$$

where

$$
\left(\begin{array}{l}
n \\
l
\end{array}\right)=\frac{n(n-1) \cdots(n-l+1)}{l !} .
$$

The recurrence formula of $B_{n}$ is given by

$$
B_{0}=1, \quad(B+1)^{n}-B_{n}= \begin{cases}1, & n=1, \\ 0, & n>1,\end{cases}
$$

with the usual convention of denoting $B^{n}$ by $B_{n}$.

By (2.6) and (2.8), we have

$$
B_{0}=1, \quad B_{n}(1)-B_{n}=\delta_{1, n} \quad \text { if } n \in \mathbb{N} .
$$

The fermionic $p$-adic integral $I_{-1}(f)$ on $\mathbb{Z}_{p}$ is defined by

$$
I_{-1}(f(z))=\int_{\mathbb{Z}_{p}} f(z) d \mu_{-1}(z)=\lim _{N \rightarrow \infty} \sum_{a=0}^{p^{N}-1} f(a)(-1)^{a}
$$

where $f \in U D\left(\mathbb{Z}_{p}\right)$. This integral was introduced by Kim [12] in order to derive useful formulas involving the Euler numbers and polynomials. It has also been defined independently by Shiratani and Yamamoto [31] in order to interpolate the Euler numbers 
$p$-adically. Osipov [32] gave a new proof of the existence of the Kubota-Leopoldt $p$-adic zeta function by using the integral representation

$$
I_{\varepsilon}(f(z))=\int_{\mathbb{Z}_{p}} f(z) d \mu_{\varepsilon}(z)=\lim _{N \rightarrow \infty} \sum_{a=0}^{p^{I N}-1} f(a) \varepsilon^{a},
$$

where $f \in U D\left(\mathbb{Z}_{p}\right), \varepsilon^{k}=1, \varepsilon \neq 1,(k, p)=1$, and $k \mid\left(p^{l}-1\right)$. Note that when $k=2, I_{\varepsilon}(f)$ is the fermionic $p$-adic integral $I_{-1}(f)$ on $\mathbb{Z}_{p}$. Recently, the fermionic $p$-adic integral $I_{-1}(f)$ on $\mathbb{Z}_{p}$ has been used by the third author to give a brief proof of Stein's classical result on Euler numbers modulo power of two [10]; it has also been used by Maïga [33] to give some new identities and congruences concerning Euler numbers and polynomials.

From the definition of $I_{-1}(f)$, we have

$$
I_{-1}\left(f_{1}\right)+I_{-1}(f)=2 f(0) \text {, }
$$

where $f_{1}(z)=f(z+1)$ (see [12, Lemma 1]). Thus from induction, we have the following fermionic $p$-adic integral equation:

$$
I_{-1}\left(f_{\alpha}\right)+(-1)^{\alpha-1} I_{-1}(f)=2 \sum_{i=0}^{\alpha-1}(-1)^{\alpha-i-1} f(i),
$$

where $\alpha \in \mathbb{N}$ and

$$
f_{\alpha}(z)=f(z+\alpha)
$$

(see [12, Theorem 2]).

Using formula (2.12), we have the following fermionic $p$-adic integral representation of the generating function of Euler polynomials $E_{n}(x)$ :

$$
I_{-1}\left(e^{(x+z) t}\right)=\frac{2}{e^{t}+1} e^{x t}=\sum_{n=0}^{\infty} E_{n}(x) \frac{t^{n}}{n !}
$$

namely

$$
I_{-1}\left((x+z)^{n}\right)=E_{n}(x)
$$

(see [34, Proposition 2.1]).

Setting $x=0$ in (2.16), we obtain the fermionic $p$-adic integral representation of the $n$th Euler numbers

$$
E_{n}=E_{n}(0)=I_{-1}\left(z^{n}\right)
$$

(comparing with (2.5)).

Therefore, by the binomial theorem, (2.16), and (2.17), we have

$$
E_{n}(x)=\sum_{l=0}^{n}\left(\begin{array}{l}
n \\
l
\end{array}\right) x^{l} I_{-1}\left(z^{n-l}\right)=\sum_{l=0}^{n}\left(\begin{array}{l}
n \\
l
\end{array}\right) x^{l} E_{n-l} .
$$


The recurrence formula of $E_{n}$ is given by

$$
E_{0}=1, \quad(E+1)^{n}+E_{n}=0 \quad \text { if } n \in \mathbb{N},
$$

with the usual convention of denoting $E^{n}$ by $E_{n}$.

By (2.18) and (2.19), we have

$$
E_{n}(1)+E_{n}=2 \delta_{0, n} \quad \text { if } n \in \mathbb{N}_{0}
$$

The $p$-adic integral also implies the following reflection symmetric relations for the Bernoulli and the Euler polynomials (see formulas (2.12) and (2.13) in [34]):

$$
\begin{aligned}
& B_{n}(x)=(-1)^{n} B_{n}(1-x), \quad \text { where } n \in \mathbb{N}_{0}, \\
& E_{n}(x)=(-1)^{n} E_{n}(1-x), \quad \text { where } n \in \mathbb{N}_{0} .
\end{aligned}
$$

\section{Identities involving Bernoulli and Euler numbers}

In this section, we prove new identities involving Bernoulli and Euler numbers.

Lemma 3.1 For $l \in \mathbb{N}_{0}$, we have

$$
I_{-1}\left((1-x)^{l}\right)=2+E_{l}-2 \delta_{0, l}
$$

Proof Putting $f(x)=(1-x)^{l}$ in $(2.12)$, by (2.17), we have

$$
I_{-1}\left((1-x)^{l}\right)=2-I_{-1}\left((-x)^{l}\right)=2+(-1)^{l+1} E_{l} .
$$

By (2.22), with $x=0$, and (2.20), we obtain

$$
(-1)^{l} E_{l}=(-1)^{l} E_{l}(0)=E_{l}(1)=2 \delta_{0, l}-E_{l}
$$

substituting it to (3.1), we have the desired result.

The following well-known fact on Euler polynomials may also be established using $p$-adic integral. We refer to Corollary 1.1 in [35] for another proof.

Theorem $3.2 E_{2 n}=0$ for $n \geq 1$.

Proof We prove our result following the method of the proof for Theorem 2.1 in [8].

By (2.17) and (2.18), we have

$$
I_{-1}\left(E_{n}(x)\right)=\sum_{l=0}^{n}\left(\begin{array}{l}
n \\
l
\end{array}\right) E_{n-l} I_{-1}\left(x^{l}\right)=\sum_{l=0}^{n}\left(\begin{array}{l}
n \\
l
\end{array}\right) E_{n-l} E_{l} .
$$

On the other hand, using (2.18), (2.22) and Lemma 3.1, we obtain

$$
\begin{aligned}
I_{-1}\left(E_{n}(x)\right) & =(-1)^{n} I_{-1}\left(E_{n}(1-x)\right) \\
& =(-1)^{n} \sum_{l=0}^{n}\left(\begin{array}{l}
n \\
l
\end{array}\right) E_{n-l} I_{-1}\left((1-x)^{l}\right)
\end{aligned}
$$




$$
\begin{aligned}
& =(-1)^{n} \sum_{l=0}^{n}\left(\begin{array}{l}
n \\
l
\end{array}\right) E_{n-l}\left(2+E_{l}-2 \delta_{0, l}\right) \\
& =(-1)^{n} 2\left(\sum_{l=0}^{n}\left(\begin{array}{l}
n \\
l
\end{array}\right) E_{n-l}-E_{n}\right)+(-1)^{n} \sum_{l=0}^{n}\left(\begin{array}{l}
n \\
l
\end{array}\right) E_{n-l} E_{l},
\end{aligned}
$$

where $\delta_{0, l}$ is the Kronecker symbol.

Comparing (3.2) and (3.3), then using (2.18), with $x=1$, and (2.20), we have

$$
\begin{aligned}
\left(1+(-1)^{n+1}\right) \sum_{l=0}^{n}\left(\begin{array}{l}
n \\
l
\end{array}\right) E_{n-l} E_{l} & =(-1)^{n} 2\left(\sum_{l=0}^{n}\left(\begin{array}{l}
n \\
l
\end{array}\right) E_{n-l}-E_{n}\right) \\
& =(-1)^{n} 2\left(E_{n}(1)-E_{n}\right) \\
& =(-1)^{n} 4\left(\delta_{0, n}-E_{n}\right) \quad \text { if } n \in \mathbb{N}_{0} .
\end{aligned}
$$

Putting $n=0$ on the left-hand side of (3.4), we have $0=1-E_{0}$, hence $E_{0}=1$. Replacing $n$ by $2 n(n \geq 1)$, the right-hand side becomes $E_{2 n}=0$, which completes the proof.

Theorem 3.3 For $n \in \mathbb{N}_{0}$, we have

$$
\sum_{l=0}^{2 n+1}\left(\begin{array}{c}
2 n+1 \\
l
\end{array}\right) B_{2 n-l+1} E_{l}=-\delta_{0, n}
$$

Proof The proof goes the same way as in the above theorem.

By (2.5) and (2.6), we have

$$
I_{-1}\left(B_{n}(x)\right)=\sum_{l=0}^{n}\left(\begin{array}{l}
n \\
l
\end{array}\right) B_{n-l} I_{-1}\left(x^{l}\right)=\sum_{l=0}^{n}\left(\begin{array}{l}
n \\
l
\end{array}\right) B_{n-l} E_{l} .
$$

On the other hand, using (2.6), (2.21) and Lemma 3.1, we obtain

$$
\begin{aligned}
I_{-1}\left(B_{n}(x)\right) & =(-1)^{n} I_{-1}\left(B_{n}(1-x)\right) \\
& =(-1)^{n} \sum_{l=0}^{n}\left(\begin{array}{l}
n \\
l
\end{array}\right) B_{n-l} I_{-1}\left((1-x)^{l}\right) \\
& =(-1)^{n} \sum_{l=0}^{n}\left(\begin{array}{l}
n \\
l
\end{array}\right) B_{n-l}\left(2+E_{l}-2 \delta_{0, l}\right) \\
& =(-1)^{n} 2\left(\sum_{l=0}^{n}\left(\begin{array}{l}
n \\
l
\end{array}\right) B_{n-l}-B_{n}\right)+(-1)^{n} \sum_{l=0}^{n}\left(\begin{array}{l}
n \\
l
\end{array}\right) B_{n-l} E_{l} .
\end{aligned}
$$

Comparing (3.5) and (3.6), then using (2.6), with $x=1$, and (2.9), we have

$$
\begin{aligned}
\left(1+(-1)^{n+1}\right) \sum_{l=0}^{n}\left(\begin{array}{l}
n \\
l
\end{array}\right) B_{n-l} E_{l} & =(-1)^{n} 2\left(\sum_{l=0}^{n}\left(\begin{array}{l}
n \\
l
\end{array}\right) B_{n-l}-B_{n}\right) \\
& =(-1)^{n} 2\left(B_{n}(1)-B_{n}\right) \\
& =(-1)^{n} 2 \delta_{1, n} .
\end{aligned}
$$

Letting $n=2 m+1(m \geq 0)$ in this identity, we get our result. 
Theorem 3.4 For $m, n \in \mathbb{N}_{0}$, we have

$$
\begin{aligned}
& \sum_{k=0}^{2 m+1} \sum_{l=0}^{2 n+2}\left(\begin{array}{c}
2 m+1 \\
k
\end{array}\right)\left(\begin{array}{c}
2 n+2 \\
l
\end{array}\right) B_{2 m-k+1} E_{2 n-l+2} E_{k+l}=0, \\
& \sum_{k=0}^{2 m+2} \sum_{l=0}^{2 n+1}\left(\begin{array}{c}
2 m+2 \\
k
\end{array}\right)\left(\begin{array}{c}
2 n+1 \\
l
\end{array}\right) B_{2 m-k+2} E_{2 n-l+1} E_{k+l}=2 B_{2 m+2} E_{2 n+1} .
\end{aligned}
$$

Taking $m=0, n=0$ in (3.7) and (3.8), respectively, we obtain the following corollary.

Corollary 3.5 For $m, n \in \mathbb{N}_{0}$, we have

$$
\begin{aligned}
& \sum_{l=0}^{2 n+2}\left(\begin{array}{c}
2 n+2 \\
l
\end{array}\right) E_{2 n-l+2}\left(E_{l}-2 E_{l+1}\right)=0, \\
& \sum_{k=0}^{2 m+1}\left(\begin{array}{c}
2 m+1 \\
k
\end{array}\right) B_{2 m-k+1}\left(E_{k+2}-E_{k+1}\right)=0, \\
& \sum_{k=0}^{2 m}\left(\begin{array}{c}
2 m \\
k
\end{array}\right) B_{2 m-k}\left(E_{k}-2 E_{k+1}\right)=2 B_{2 m}, \\
& \sum_{l=0}^{2 n+1}\left(\begin{array}{c}
2 n+1 \\
l
\end{array}\right) E_{2 n-l+1}\left(E_{l}-6 E_{l+1}+6 E_{l+2}\right)=2 E_{2 n+1} .
\end{aligned}
$$

Proof of Theorem 3.4 We prove our result following the method of the proof for Theorem 2.3 in [8].

Putting $x=1$ in (2.6), (2.18), and using (2.9), (2.20), we get

$$
\begin{aligned}
& B_{n}(1)=\sum_{l=0}^{n}\left(\begin{array}{l}
n \\
l
\end{array}\right) B_{n-l}=B_{n}+\delta_{1, n}, \\
& E_{n}(1)=\sum_{l=0}^{n}\left(\begin{array}{l}
n \\
l
\end{array}\right) E_{n-l}=2 \delta_{0, n}-E_{n} .
\end{aligned}
$$

By the linear property of $p$-adic integral and (2.17), we have the following fermionic $p$-adic integral representation of the product of Bernoulli and Euler polynomials:

$$
\begin{aligned}
I_{-1}\left(B_{m}(x) E_{n}(x)\right) & =\sum_{k=0}^{m} \sum_{l=0}^{n}\left(\begin{array}{l}
m \\
k
\end{array}\right)\left(\begin{array}{l}
n \\
l
\end{array}\right) B_{m-k} E_{n-l} I_{-1}\left(x^{k+l}\right) \\
& =\sum_{k=0}^{m} \sum_{l=0}^{n}\left(\begin{array}{l}
m \\
k
\end{array}\right)\left(\begin{array}{l}
n \\
l
\end{array}\right) B_{m-k} E_{n-l} E_{k+l} .
\end{aligned}
$$

Further, by (2.21), (2.22), Lemma 3.1 and (3.13), we have

$$
\begin{aligned}
I_{-1}\left(B_{m}(x) E_{n}(x)\right) & =I_{-1}\left((-1)^{m+n} B_{m}(1-x) E_{n}(1-x)\right) \\
& =(-1)^{m+n} \sum_{k=0}^{m} \sum_{l=0}^{n}\left(\begin{array}{c}
m \\
k
\end{array}\right)\left(\begin{array}{l}
n \\
l
\end{array}\right) B_{m-k} E_{n-l} I_{-1}\left((1-x)^{k+l}\right)
\end{aligned}
$$




$$
\begin{aligned}
= & (-1)^{m+n} \sum_{k=0}^{m} \sum_{l=0}^{n}\left(\begin{array}{l}
m \\
k
\end{array}\right)\left(\begin{array}{l}
n \\
l
\end{array}\right) B_{m-k} E_{n-l}\left(2+E_{k+l}-2 \delta_{0, k+l}\right) \\
= & (-1)^{m+n} 2 \sum_{k=0}^{m} \sum_{l=0}^{n}\left(\begin{array}{l}
m \\
k
\end{array}\right)\left(\begin{array}{l}
n \\
l
\end{array}\right) B_{m-k} E_{n-l}+(-1)^{m+n+1} 2 B_{m} E_{n} \\
& +(-1)^{m+n} \sum_{k=0}^{m} \sum_{l=0}^{n}\left(\begin{array}{l}
m \\
k
\end{array}\right)\left(\begin{array}{l}
n \\
l
\end{array}\right) B_{m-k} E_{n-l} E_{k+l} \\
= & (-1)^{m+n} 2\left(\sum_{k=0}^{m}\left(\begin{array}{l}
m \\
k
\end{array}\right) B_{m-k}\right)\left(\sum_{l=0}^{n}\left(\begin{array}{l}
n \\
l
\end{array}\right) E_{n-l}\right)+(-1)^{m+n+1} 2 B_{m} E_{n} \\
& +(-1)^{m+n} \sum_{k=0}^{m} \sum_{l=0}^{n}\left(\begin{array}{l}
m \\
k
\end{array}\right)\left(\begin{array}{l}
n \\
l
\end{array}\right) B_{m-k} E_{n-l} E_{k+l} \\
= & (-1)^{m+n} 2 B_{m}(1) E_{n}(1)+(-1)^{m+n+1} 2 B_{m} E_{n} \\
& +(-1)^{m+n} \sum_{k=0}^{m} \sum_{l=0}^{n}\left(\begin{array}{l}
m \\
k
\end{array}\right)\left(\begin{array}{l}
n \\
l
\end{array}\right) B_{m-k} E_{n-l} E_{k+l} .
\end{aligned}
$$

Comparing (3.14) and (3.15), then using (3.13), we have

$$
\begin{aligned}
(1 & \left.+(-1)^{m+n+1}\right) \sum_{k=0}^{m} \sum_{l=0}^{n}\left(\begin{array}{l}
m \\
k
\end{array}\right)\left(\begin{array}{l}
n \\
l
\end{array}\right) B_{m-k} E_{n-l} E_{k+l} \\
& =(-1)^{m+n} 2 B_{m}(1) E_{n}(1)+(-1)^{m+n+1} 2 B_{m} E_{n} \\
& =(-1)^{m+n} 2\left(B_{m}+\delta_{1, m}\right)\left(2 \delta_{0, n}-E_{n}\right)+(-1)^{m+n+1} 2 B_{m} E_{n} \\
& =(-1)^{m+n} 2\left(2 B_{m} \delta_{0, n}-2 B_{m} E_{n}+2 \delta_{1, m} \delta_{0, n}-E_{n} \delta_{1, m}\right) .
\end{aligned}
$$

Replacing $m$ by $2 m+1$ and $n$ by $2 n+1$ in (3.16), respectively, we have

$$
\begin{aligned}
(1+ & \left.(-1)^{n}\right) \sum_{k=0}^{2 m+1} \sum_{l=0}^{n}\left(\begin{array}{c}
2 m+1 \\
k
\end{array}\right)\left(\begin{array}{l}
n \\
l
\end{array}\right) B_{2 m-k+1} E_{n-l} E_{k+l} \\
& =(-1)^{n+1} 2\left(2 B_{2 m+1} \delta_{0, n}-2 B_{2 m+1} E_{n}+2 \delta_{1,2 m+1} \delta_{0, n}-E_{n} \delta_{1,2 m+1}\right)
\end{aligned}
$$

and

$$
\begin{aligned}
(1+ & \left.(-1)^{m}\right) \sum_{k=0}^{m} \sum_{l=0}^{2 n+1}\left(\begin{array}{c}
m \\
k
\end{array}\right)\left(\begin{array}{c}
2 n+1 \\
l
\end{array}\right) B_{m-k} E_{2 n-l+1} E_{k+l} \\
& =(-1)^{m} 2\left(2 B_{m} E_{2 n+1}+E_{2 n+1} \delta_{1, m}\right) .
\end{aligned}
$$

Finally, replacing $n$ by $2 n+2$ in (3.17) and $m$ by $2 m$ in (3.18), using Theorem 3.2, we get our result.

Remark 3.6 Putting $n=0$ in (3.17), we recover Theorem 3.3. Setting $m=0$ in (3.18), we obtain

$$
\sum_{l=0}^{2 n+1}\left(\begin{array}{c}
2 n+1 \\
l
\end{array}\right) E_{2 n-l+1} E_{l}=E_{2 n+1} E_{0}+\underbrace{E_{2 n} E_{1}+\cdots+E_{1} E_{2 n}}_{(2 n-1) \text {-terms }}+E_{0} E_{2 n+1}=2 E_{2 n+1} \quad \text { if } n \in \mathbb{N} \text {. }
$$


This identity is trivial, because by Theorem 3.2 , the $(2 n-1)$ th terms are identical to zero.

\section{Identities involving higher-order Bernoulli and Euler numbers}

In this section, we prove new identities involving higher-order Bernoulli and Euler numbers and polynomials.

The higher-order Bernoulli polynomials $B_{n}^{(\alpha)}(x)$ and the higher-order Euler polynomials $E_{n}^{(\alpha)}(x)$ are defined by the following generating functions:

$$
\left(\frac{t}{e^{t}-1}\right)^{\alpha} e^{x t}=\sum_{n=0}^{\infty} B_{n}^{(\alpha)}(x) \frac{t^{n}}{n !}
$$

and

$$
\left(\frac{2}{e^{t}+1}\right)^{\alpha} e^{x t}=\sum_{n=0}^{\infty} E_{n}^{(\alpha)}(x) \frac{t^{n}}{n !}
$$

respectively. For $n \in \mathbb{N}_{0}$, we have $B_{n}^{(\alpha)}=B_{n}^{(\alpha)}(0)$ and $E_{n}^{(\alpha)}=E_{n}^{(\alpha)}(0)$, where $B_{n}^{(\alpha)}$ and $E_{n}^{(\alpha)}$ denote the higher-order Bernoulli and Euler numbers, respectively (see [20, Section 1.6] and [21, (16), (17)]). Moreover, from (4.1) and (4.2), we have

$$
\begin{aligned}
& B_{n}^{(\alpha)}(x)=\sum_{l=0}^{n}\left(\begin{array}{l}
n \\
l
\end{array}\right) B_{n-l}^{(\alpha)} x^{l}, \\
& B_{n}^{(\alpha)}(x)=(-1)^{n} B_{n}^{(\alpha)}(\alpha-x), \\
& E_{n}^{(\alpha)}(x)=\sum_{l=0}^{n}\left(\begin{array}{l}
n \\
l
\end{array}\right) E_{n-l}^{(\alpha)} x^{l}, \\
& E_{n}^{(\alpha)}(x)=(-1)^{n} E_{n}^{(\alpha)}(\alpha-x) .
\end{aligned}
$$

Lemma 4.1 For $\alpha \in \mathbb{N}$ and $l \in \mathbb{N}_{0}$, we have

$$
I_{-1}\left((\alpha-x)^{l}\right)=(-1)^{\alpha}\left(2 \delta_{0, l}-E_{l}\right)+2 \sum_{i=0}^{\alpha-1}(-1)^{i}(\alpha-i)^{l} .
$$

Proof Letting $f(x)=(\alpha-x)^{l}(\alpha \in \mathbb{N})$ in (2.14), we have $f_{\alpha}(x)=(-x)^{l}$.

By (2.13), we have

$$
\begin{aligned}
I_{-1}\left((\alpha-x)^{l}\right) & =(-1)^{\alpha} I_{-1}\left((-x)^{l}\right)+2 \sum_{i=0}^{\alpha-1}(-1)^{-i} f(i) \\
& =(-1)^{\alpha}(-1)^{l} E_{l}+2 \sum_{i=0}^{\alpha-1}(-1)^{i}(\alpha-i)^{l} .
\end{aligned}
$$

By (2.20), (2.22) with $x=0$, we have $(-1)^{l} E_{l}=E_{l}(1)=2 \delta_{0, l}-E_{l}$, and we obtain the assertion of the lemma. 
Theorem 4.2 For $\alpha \in \mathbb{N}$ and $n \in \mathbb{N}_{0}$, we have

$$
\sum_{i=0}^{\alpha}(-1)^{i} B_{n}^{(\alpha)}(i)=\left\{\begin{array}{lll}
\sum_{l=0}^{n}\left(\begin{array}{c}
n \\
l
\end{array}\right) B_{n-l}^{(\alpha)} E_{l} & \text { if } n+\alpha \equiv 0 & (\bmod 2) \\
0 & \text { if } n+\alpha \neq 00 & (\bmod 2)
\end{array}\right.
$$

Proof From (2.17), (4.3) and the linear property of $p$-adic integral, we obtain the following fermionic $p$-adic integral representation of the higher-order Bernoulli polynomials:

$$
I_{-1}\left(B_{n}^{(\alpha)}(x)\right)=\sum_{l=0}^{n}\left(\begin{array}{l}
n \\
l
\end{array}\right) B_{n-l}^{(\alpha)} I_{-1}\left(x^{l}\right)=\sum_{l=0}^{n}\left(\begin{array}{l}
n \\
l
\end{array}\right) B_{n-l}^{(\alpha)} E_{l} .
$$

On the other hand, by (4.4) and Lemma 4.1, we have

$$
\begin{aligned}
I_{-1}\left(B_{n}^{(\alpha)}(x)\right)= & (-1)^{n} \sum_{l=0}^{n}\left(\begin{array}{l}
n \\
l
\end{array}\right) B_{n-l}^{(\alpha)} I_{-1}\left((\alpha-x)^{l}\right) \\
= & (-1)^{n} \sum_{l=0}^{n}\left(\begin{array}{l}
n \\
l
\end{array}\right) B_{n-l}^{(\alpha)}\left((-1)^{\alpha}\left(2 \delta_{0, l}-E_{l}\right)+2 \sum_{i=0}^{\alpha-1}(-1)^{i}(\alpha-i)^{l}\right) \\
= & (-1)^{n+\alpha} 2 B_{n}^{(\alpha)}+(-1)^{n} 2 \sum_{l=0}^{n}\left(\begin{array}{l}
n \\
l
\end{array}\right) B_{n-l}^{(\alpha)} \sum_{i=0}^{\alpha-1}(-1)^{i}(\alpha-i)^{l} \\
& -(-1)^{n+\alpha} \sum_{l=0}^{n}\left(\begin{array}{l}
n \\
l
\end{array}\right) B_{n-l}^{(\alpha)} E_{l} .
\end{aligned}
$$

Clearly,

$$
\sum_{i=0}^{\alpha-1}(-1)^{i}(\alpha-i)^{l}=\sum_{i=1}^{\alpha}(-1)^{\alpha-i} i^{l} .
$$

Comparing (4.8) and (4.9), then using (4.10) and (4.3) with $x=i$, we obtain

$$
\begin{aligned}
(1 & \left.+(-1)^{n+\alpha}\right) \sum_{l=0}^{n}\left(\begin{array}{l}
n \\
l
\end{array}\right) B_{n-l}^{(\alpha)} E_{l} \\
& =(-1)^{n+\alpha} 2 B_{n}^{(\alpha)}+(-1)^{n} 2 \sum_{l=0}^{n}\left(\begin{array}{l}
n \\
l
\end{array}\right) B_{n-l}^{(\alpha)} \sum_{i=0}^{\alpha-1}(-1)^{i}(\alpha-i)^{l} \\
& =(-1)^{n+\alpha} 2 B_{n}^{(\alpha)}+(-1)^{n} 2 \sum_{l=0}^{n}\left(\begin{array}{l}
n \\
l
\end{array}\right) B_{n-l}^{(\alpha)} \sum_{i=1}^{\alpha}(-1)^{\alpha-i} i^{l} \\
& =(-1)^{n+\alpha} 2 B_{n}^{(\alpha)}+(-1)^{n} 2 \sum_{i=1}^{\alpha}(-1)^{\alpha-i}\left(\sum_{l=0}^{n}\left(\begin{array}{l}
n \\
l
\end{array}\right) B_{n-l}^{(\alpha)} l^{l}\right) \\
& =(-1)^{n+\alpha} 2 B_{n}^{(\alpha)}+(-1)^{n} 2 \sum_{i=1}^{\alpha}(-1)^{\alpha-i} B_{n}^{(\alpha)}(i) \\
& =(-1)^{n+\alpha} 2\left(B_{n}^{(\alpha)}+\sum_{i=1}^{\alpha}(-1)^{i} B_{n}^{(\alpha)}(i)\right) .
\end{aligned}
$$


Since $B_{n}^{(\alpha)}=B_{n}^{(\alpha)}(0)$, therefore, by (4.11), we have

$$
(-1)^{n+\alpha} 2 \sum_{i=0}^{\alpha}(-1)^{i} B_{n}^{(\alpha)}(i)=\left(1+(-1)^{n+\alpha}\right) \sum_{l=0}^{n}\left(\begin{array}{l}
n \\
l
\end{array}\right) B_{n-l}^{(\alpha)} E_{l},
$$

which is the required result.

Theorem 4.3 For $\alpha \in \mathbb{N}$ and $n \in \mathbb{N}_{0}$, we have

$$
\sum_{i=0}^{\alpha}(-1)^{i} E_{n}^{(\alpha)}(i)=\left\{\begin{array}{lll}
\sum_{l=0}^{n}\left(\begin{array}{l}
n \\
l
\end{array}\right) E_{n-l}^{(\alpha)} E_{l} & \text { if } n+\alpha \equiv 0 & (\bmod 2) \\
0 & \text { if } n+\alpha \neq 0 & (\bmod 2)
\end{array}\right.
$$

Proof This follows from the same process as in the proof of Theorem 4.2 by using (2.17), (4.5), (4.6) and Lemma 4.1.

Theorem 4.4 For $m, n \in \mathbb{N}_{0}$ and $\alpha \in \mathbb{N}$, we have

$$
\begin{aligned}
& \sum_{i=0}^{\alpha}(-1)^{i} B_{m}^{(\alpha)}(i) E_{n}^{(\alpha)}(i) \\
& \quad=\left\{\begin{array}{lll}
\sum_{k=0}^{m} \sum_{l=0}^{n}\left(\begin{array}{c}
m \\
k
\end{array}\right)\left(\begin{array}{l}
n \\
l
\end{array}\right) B_{m-k}^{(\alpha)} E_{n-l}^{(\alpha)} E_{k+l} & \text { if } m+n+\alpha \equiv 0 & (\bmod 2), \\
0 & \text { if } m+n+\alpha \neq 0 & (\bmod 2) .
\end{array}\right.
\end{aligned}
$$

Proof First we have the following fermionic $p$-adic integral representation of the product of higher-order Bernoulli and Euler polynomials:

$$
\begin{aligned}
I_{-1}\left(B_{m}^{(\alpha)}(x) E_{n}^{(\alpha)}(x)\right) & =\sum_{k=0}^{m} \sum_{l=0}^{n}\left(\begin{array}{c}
m \\
k
\end{array}\right)\left(\begin{array}{c}
n \\
l
\end{array}\right) B_{m-k}^{(\alpha)} E_{n-l}^{(\alpha)} I_{-1}\left(x^{k+l}\right) \\
& =\sum_{k=0}^{m} \sum_{l=0}^{n}\left(\begin{array}{c}
m \\
k
\end{array}\right)\left(\begin{array}{c}
n \\
l
\end{array}\right) B_{m-k}^{(\alpha)} E_{n-l}^{(\alpha)} E_{k+l} .
\end{aligned}
$$

On the other hand, by (4.4), (4.6), Lemma 4.1, by (4.3) and (4.5) with $x=i$, we have

$$
\begin{aligned}
I_{-1}( & \left.B_{m}^{(\alpha)}(x) E_{n}^{(\alpha)}(x)\right) \\
= & I_{-1}\left((-1)^{m+n} B_{m}^{(\alpha)}(1-x) E_{n}^{(\alpha)}(1-x)\right) \\
= & (-1)^{m+n} \sum_{k=0}^{m} \sum_{l=0}^{n}\left(\begin{array}{c}
m \\
k
\end{array}\right)\left(\begin{array}{c}
n \\
l
\end{array}\right) B_{m-k}^{(\alpha)} E_{n-l}^{(\alpha)} I_{-1}\left((\alpha-x)^{k+l}\right) \\
= & (-1)^{m+n+\alpha} \sum_{k=0}^{m} \sum_{l=0}^{n}\left(\begin{array}{c}
m \\
k
\end{array}\right)\left(\begin{array}{c}
n \\
l
\end{array}\right) B_{m-k}^{(\alpha)} E_{n-l}^{(\alpha)}\left(2 \delta_{0, k+l}-E_{k+l}+2 \sum_{i=1}^{\alpha}(-1)^{i} i^{k+l}\right) \\
= & (-1)^{m+n+\alpha} 2\left(B_{m}^{(\alpha)} E_{n}^{(\alpha)}+\sum_{i=1}^{\alpha}(-1)^{i} \sum_{k=0}^{m} \sum_{l=0}^{n}\left(\begin{array}{c}
m \\
k
\end{array}\right)\left(\begin{array}{c}
n \\
l
\end{array}\right) B_{m-k}^{(\alpha)} i^{k} E_{n-l}^{(\alpha)} l^{l}\right) \\
& \quad-(-1)^{m+n+\alpha} \sum_{k=0}^{m} \sum_{l=0}^{n}\left(\begin{array}{c}
m \\
k
\end{array}\right)\left(\begin{array}{c}
n \\
l
\end{array}\right) B_{m-k}^{(\alpha)} E_{n-l}^{(\alpha)} E_{k+l}
\end{aligned}
$$




$$
\begin{aligned}
= & (-1)^{m+n+\alpha} 2\left(B_{m}^{(\alpha)} E_{n}^{(\alpha)}+\sum_{i=1}^{\alpha}(-1)^{i}\left(\sum_{k=0}^{m}\left(\begin{array}{c}
m \\
k
\end{array}\right) B_{m-k}^{(\alpha)} i^{k}\right)\left(\sum_{l=0}^{n}\left(\begin{array}{c}
n \\
l
\end{array}\right) E_{n-l}^{(\alpha)} i^{l}\right)\right) \\
& -(-1)^{m+n+\alpha} \sum_{k=0}^{m} \sum_{l=0}^{n}\left(\begin{array}{c}
m \\
k
\end{array}\right)\left(\begin{array}{c}
n \\
l
\end{array}\right) B_{m-k}^{(\alpha)} E_{n-l}^{(\alpha)} E_{k+l} \\
= & (-1)^{m+n+\alpha} 2\left(B_{m}^{(\alpha)} E_{n}^{(\alpha)}+\sum_{i=1}^{\alpha}(-1)^{i} B_{m}^{(\alpha)}(i) E_{n}^{(\alpha)}(i)\right) \\
& -(-1)^{m+n+\alpha} \sum_{k=0}^{m} \sum_{l=0}^{n}\left(\begin{array}{c}
m \\
k
\end{array}\right)\left(\begin{array}{c}
n \\
l
\end{array}\right) B_{m-k}^{(\alpha)} E_{n-l}^{(\alpha)} E_{k+l} .
\end{aligned}
$$

Comparing (4.12) and (4.13), and also noticing that $B_{n}^{(\alpha)}=B_{n}^{(\alpha)}(0)$ and $E_{n}^{(\alpha)}=E_{n}^{(\alpha)}(0)$, we have

$$
\begin{aligned}
(1+ & \left.(-1)^{m+n+\alpha}\right) \sum_{k=0}^{m} \sum_{l=0}^{n}\left(\begin{array}{c}
m \\
k
\end{array}\right)\left(\begin{array}{l}
n \\
l
\end{array}\right) B_{m-k}^{(\alpha)} E_{n-l}^{(\alpha)} E_{k+l} \\
& =(-1)^{m+n+\alpha} 2 \sum_{i=0}^{\alpha}(-1)^{i} B_{m}^{(\alpha)}(i) E_{n}^{(\alpha)}(i),
\end{aligned}
$$

which is the assertion of the theorem.

\section{Further remarks and observations}

In this section, we show that the same methods as in Sections 3 and 4 can be used to obtain a new identity involving both Bernoulli, Euler and Genocchi numbers.

The higher-order Genocchi polynomials $G_{n}^{(\alpha)}(x)$ are defined by the generating function

$$
\left(\frac{2 t}{e^{t}+1}\right)^{\alpha} e^{x t}=\sum_{n=0}^{\infty} G_{n}^{(\alpha)}(x) \frac{t^{n}}{n !} .
$$

The Genocchi polynomials $G_{n}(x)$ are given by $G_{n}(x)=G_{n}^{(1)}(x)$. For $x=0$, we have the Genocchi numbers $G_{n}$, i.e., $G_{n}=G_{n}(0)$. Letting $x=0$, we also have $G_{n}^{(\alpha)}=G_{n}^{(\alpha)}(0)$, where $G_{n}^{(\alpha)}$ denotes the higher-order Genocchi numbers.

The generating function of Genocchi polynomials is similar to those of Bernoulli and Euler polynomials, so it may be expected that the Genocchi numbers also satisfy similar identities as those established in Sections 3 and 4.

From the generating function (5.1), it is easy to deduce that

$$
\begin{aligned}
& G_{n}^{(\alpha)}(x)=\sum_{l=0}^{n}\left(\begin{array}{l}
n \\
l
\end{array}\right) G_{n-l}^{(\alpha)} x^{l}, \\
& G_{n}^{(\alpha)}(x)=(-1)^{n+\alpha} G_{n}^{(\alpha)}(\alpha-x) .
\end{aligned}
$$

Therefore, by the fermionic $p$-adic integral representation of $G_{n}^{(\alpha)}(x),(5.2)$ and (5.3), we have

$$
\left(1+(-1)^{n}\right) \sum_{l=0}^{n}\left(\begin{array}{l}
n \\
l
\end{array}\right) G_{n-l}^{(\alpha)} E_{l}=(-1)^{n} 2 \sum_{i=0}^{\alpha}(-1)^{i} G_{n}^{(\alpha)}(i) .
$$


For $n \not \equiv 0(\bmod 2)$, by $(5.4)$, we have $\sum_{i=0}^{\alpha}(-1)^{i} G_{n}^{(\alpha)}(i)=0$. From $(5.4)$, it is easily seen that

$$
\sum_{l=0}^{2 n}\left(\begin{array}{c}
2 n \\
l
\end{array}\right) G_{2 n-l}^{(\alpha)} E_{l}=\sum_{i=0}^{\alpha}(-1)^{i} G_{2 n}^{(\alpha)}(i), \quad n \in \mathbb{N} .
$$

Also, by the fermionic $p$-adic representations of $B_{n}^{(\alpha)}(x)$ and $G_{n}^{(\alpha)}(x)$, we have

$$
\begin{aligned}
\left(1+(-1)^{m+n}\right) \sum_{k=0}^{m} \sum_{l=0}^{n}\left(\begin{array}{c}
m \\
k
\end{array}\right)\left(\begin{array}{l}
n \\
l
\end{array}\right) B_{m-k}^{(\alpha)} G_{n-l}^{(\alpha)} E_{k+l} \\
=(-1)^{m+n} 2 \sum_{i=0}^{\alpha}(-1)^{i} B_{m}^{(\alpha)}(i) G_{n}^{(\alpha)}(i),
\end{aligned}
$$

which is equivalent to

$$
\begin{aligned}
& \sum_{i=0}^{\alpha}(-1)^{i} B_{m}^{(\alpha)}(i) G_{n}^{(\alpha)}(i) \\
& \quad=\left\{\begin{array}{lll}
\sum_{k=0}^{m} \sum_{l=0}^{n}\left(\begin{array}{c}
m \\
k
\end{array}\right)\left(\begin{array}{c}
n \\
l
\end{array}\right) B_{m-k}^{(\alpha)} G_{n-l}^{(\alpha)} E_{k+l} & \text { if } m+n \equiv 0 & (\bmod 2), \\
0 & \text { if } m+n \neq 0 & (\bmod 2),
\end{array}\right.
\end{aligned}
$$

where $m, n \in \mathbb{N}_{0}$.

\section{Competing interests}

The authors declare that they have no competing interest.

\section{Authors' contributions}

All authors contributed equally in this paper. They read and approved the final manuscript.

\section{Author details}

${ }^{1}$ Department of Mathematical Sciences, Korea Advanced Institute of Science and Technology (KAIST), 373-1

Guseong-dong, Yuseong-gu, Daejeon, 305-701, Republic of Korea. ${ }^{2}$ National Institute for Mathematical Sciences, Yuseong-daero 1689-gil, Yuseong-gu, Daejeon, 305-811, Republic of Korea. ${ }^{3}$ Division of Cultural Education, Kyungnam University, 7 (Woryeong-dong) kyungnamdaehak-ro, Masanhappo-gu, Changwon-si, Gyeongsangnam-do 631-701, Republic of Korea.

\section{Acknowledgements}

This work was supported by the Kyungnam University Foundation Grant, 2013.

Received: 26 October 2012 Accepted: 5 March 2013 Published: 26 March 2013

\section{References}

1. Sitaramachandrarao, R, Davis, B: Some identities involving the Riemann zeta function II. Indian J. Pure Appl. Math. 17, 1175-1186 (1986)

2. Choi, J, Kim, DS, Kim, T, Kim, YH: A note on some identities of Frobenius-Euler numbers and polynomials. Int. J. Math. Math. Sci. 2012, Article ID 861797 (2012)

3. Dilcher, K: Sums of products of Bernoulli numbers. J. Number Theory 60, 23-41 (1996)

4. Kim, DS, Dolgy, DV, Kim, H-M, Lee, S-H, Kim, T: Integral formulae of Bernoulli polynomials. Discrete Dyn. Nat. Soc. 2012, Article ID 269847 (2012)

5. Kim, DS, Kim, T, Choi, J, Kim, YH: Some identities on Bernoulli and Euler numbers. Discrete Dyn. Nat. Soc. 2012, Article ID 486158 (2012)

6. Kim, DS, Kim, T, Choi, J, Kim, YH: Identities involving q-Bernoulli and q-Euler numbers. Abstr. Appl. Anal. 2012, Article ID $674210(2012)$

7. Kim, DS, Kim, T, Dolgy, DV, Lee, SH, Rim, S-H: Some properties and identities of Bernoulli and Euler polynomials associated with $p$-adic integral on $\mathbb{Z}_{p}$. Abstr. Appl. Anal. 2012, Article ID 847901 (2012)

8. Kim, DS, Kim, T, Lee, S-H, Dolgy, DV, Rim, S-H: Some new identities on the Bernoulli and Euler numbers. Discrete Dyn. Nat. Soc. 2011, Article ID 856132 (2011)

9. Kim, H-M, Kim, DS: Arithmetic identities involving Bernoulli and Euler numbers. Int. J. Math. Math. Sci. 2012, Article ID 689797 (2012) 
10. Kim, M-S: On Euler numbers, polynomials and related p-adic integrals. J. Number Theory 129, 2166-2179 (2009)

11. Kim, M-S: A note on sums of products of Bernoulli numbers. Appl. Math. Lett. 24(1), 55-61 (2011)

12. Kim, $\mathrm{T}$ : On the analogs of Euler numbers and polynomials associated with $p$-adic $q$-integral on $\mathbb{Z}_{p}$ at $q=-1$. J. Math. Anal. Appl. 331, 779-792 (2007)

13. Kim, T, Kim, DS, Bayad, A, Rim, S-H: Identities on the Bernoulli and the Euler numbers and polynomials. Ars Comb. CVII, 455-463 (2012)

14. Lee, I, Kim, DS: Derivation of identities involving Bernoulli and Euler numbers. Int. J. Math. Math. Sci. 2012, Article ID 598543 (2012)

15. Petojević, A: New sums of products of Bernoulli numbers. Integral Transforms Spec. Funct. 19, 105-114 (2008)

16. Petojević, A, Srivastava, HM: Computation of Euler's type sums of the products of Bernoulli numbers. Appl. Math. Lett. 22, 796-801 (2009)

17. Raabe, JL: Zurückführung einiger Summen und bestmmtiem Integrale auf die Jacob-Bernoullische Function. J. Reine Angew. Math. 42, 348-367 (1851)

18. Simsek, Y: q-analogue of twisted /-series and q-twisted Euler numbers. J. Number Theory 110, $267-278$ (2005)

19. Srivastava, HM: Some formulas for the Bernoulli and Euler polynomials at rational arguments. Math. Proc. Camb. Philos. Soc. 129, 77-84 (2000)

20. Srivastava, HM, Choi, J: Series Associated with the Zeta and Related Functions. Kluwer Academic, Dordrecht (2001)

21. Srivastava, HM, Pinter, A: Remarks on some relationships between the Bernoulli and Euler polynomials. Appl. Math. Lett. 17, 375-380 (2004)

22. Kim, M-S, Hu, S: Sums of products of Apostol-Bernoulli numbers. Ramanujan J. 28, 113-123 (2012)

23. Miki, H: A relation between Bernoulli numbers. J. Number Theory 10, 297-302 (1978)

24. Matiyasevich, Y: Identities with Bernoulli numbers. http://logic.pdmi.ras.ru/ yumat/Journal/Bernoulli/bernulli.htm

25. Dunne, GV, Schubert, C: Bernoulli number identities from quantum field theory. Preprint (2004). arXiv:math.NT/0406610

26. Crabb, MC: The Miki-Gessel Bernoulli number identity. Glasg. Math. J. 47, 327-328 (2005)

27. Gessel, IM: On Miki's identity for Bernoulli numbers. J. Number Theory 110, 75-82 (2005)

28. Sun, Z-W, Pan, H: Identities concerning Bernoulli and Euler polynomials. Acta Arith. 125, 21-39 (2006)

29. Cohen, H: Number Theory, Vol. II: Analytic and Modern Tools. Graduate Texts in Mathematics, vol. 240. Springer, New York (2007)

30. Robert, AM: A Course in p-Adic Analysis. Graduate Texts in Mathematics, vol. 198. Springer, New York (2000)

31. Shiratani, K, Yamamoto, S: On a p-adic interpolation function for the Euler numbers and its derivatives. Mem. Fac. Sci., Kyushu Univ., Ser. A, Math. 39, 113-125 (1985)

32. Osipov, JV: $p$-adic zeta functions. Usp. Mat. Nauk 34, 209-210 (1979) (in Russian)

33. Maïga, H: Some identities and congruences concerning Euler numbers and polynomials. J. Number Theory 130 , 1590-1601 (2010)

34. Kim, M-S, Hu, S: On p-adic Hurwitz-type Euler zeta functions. J. Number Theory 132, 2977-3015 (2012)

35. Sun, Z-W: Introduction to Bernoulli and Euler polynomials. A Lecture Given in Taiwan on June 6, 2002. http://math.nju.edu.cn/ zwsun/BerE.pdf

Cite this article as: Hu et al. New identities involving Bernoulli, Euler and Genocchi numbers. Advances in Difference Equations 2013 2013:74.

\section{Submit your manuscript to a SpringerOpen ${ }^{\circ}$ journal and benefit from:}

- Convenient online submission

Rigorous peer review

- Immediate publication on acceptance

- Open access: articles freely available online

- High visibility within the field

- Retaining the copyright to your article 